\title{
卫星编队用于消除海潮模型混频误差影响的 可行性研究
}

\author{
赵倩 ${ }^{(1)}$ ，姜卫平 ${ }^{(2 *}$ ，徐新禹 ${ }^{(3)}$, 邹贤才 ${ }^{(3)}$ \\ (1) 中国地震局地震预测研究所, 北京 100036 ; \\ (2) 武汉大学卫星导航定位技术研究中心, 武汉 430079; \\ (3) 武汉大学测绘学院, 武汉 430079 \\ * 联系人, E-mail: wpjiang@whu.edu.cn
}

收稿日期: 2014-03-14; 接受日期: 2014-08-08; 网络版发表日期: 2015-01-20

国家重点基础研究发展计划项目(编号：2013CB733302)、中国地震局地震预测研究所基本科研业务费专项项目(编号：2013IES0203, 2014IES010102)和国家自然科学基金青年项目(编号: 41304018)资助

\begin{abstract}
摘要目前时变信号模型的混频误差成为时变重力场解算精度的主要限制之处, 本文给出 三种适合于重力任务的包含不同方向观测量的卫星编队 GRACE-type, Pendulum-type 和 n-sCartwheel-type, 设计两种方案并通过仿真实验研究了卫星编队用于消除海潮模型混频误差影 响的可行性. 结果表明, 当不考虑模型混频误差时, n-s-Cartwheel 编队能够为重力场解算提供 最好的条件, 与 GRACE-type 编队相比, 对重力场解算精度提高达 43\%; 当海潮模型的混频误 差成为主要误差源时, 利用卫星编队由动力法反演重力场并不能消除混频及提高重力场的解 算精度, 包含径向观测量的 Cartwheel-type 编队由于对重力场的高阶变化更为敏感, 重力场结 果中包含了更多的海潮模型误差的高频信号，误差急剧增大.
\end{abstract}

关键词

卫星编队

混频误差

海潮模型

重力场反演
CHAMP, GRACE 和 GOCE 的实现，显示了空间 观测对于研究地球系统的质量变化具有极大潜力. 尤其是 GRACE 任务, 自 2002 年升空以来, 已经连续 运行 11 年, 其所获得的数据为我们研究全球海平面 变化、冰盖和冰河质量变化、深海环流及陆地水质量 迁移等方面发挥了重大作用(Wahr 等，1998，2004; Han 等, 2004; Chen 等, 2006; Tapley 等, 2004; 罗志才 等, 2012). 美国 NASA 和德国 DLR 已决定将当前的 GRACE 任务延长至 2017 年, 并计划于 2017 年发射 后续 GRACE 卫星, 以获取更长时间尺度的地球重力 场时变和静态信息, 为相关科学研究服务 (NASA,
2010, http://www.jpl.nasa.gov/mobile/news/index.cfm? release $=2010-195)$.

在 GRACE 任务初期，由于实测星间 $\mathrm{k}$ 波段测距 系统 (kbr) 数据的误差量级较大, 超过了预期的影响, 因此数据处理中其他误差的影响并没有被作为重点 考虑(Kim, 2000). 但随着空基重力测量技术的发展, 目前国际和国内的各项重力卫星新任务已经考虑到 在卫星上加载更加先进的测量设备, 如用激光测距 系统代替微波测距系统来测量星间距离变化，能将 观测精度提高 100 倍; 同时, 利用无拖曳补偿系统来 实时补偿卫星所受到的非保守力 (Kless 等, 2008;

中文引用格式: 赵倩, 姜卫平, 徐新禹, 等. 2015. 卫星编队用于消除海潮模型混频误差影响的可行性研究. 中国科学: 地球科学, 45: 169-176

英文引用格式: Zhao Q, Jiang W P, Xu X Y, et al. 2015. Feasibility study on application of satellite formations for eliminating the influence from aliasing error of ocean tide model. Science China: Earth Sciences, 58: 474-481, doi: 10.1007/s11430-014-5010-5 
Wiese等, 2009; Kurtenbach 等, 2009; Ditmar 等, 2011). 在这一趋势下, 解算时变重力场模型精度的主要限 制之处不再是传感器误差(如观测噪声), 而是模型误 差, 这些模型误差来自于大气、海流和海洋潮汐等的 质量变化. GRACE 一般使用 30 天左右的周期采样, 而一些信号如潮汐、大气、海洋和陆地水文等, 他们 的变化周期一般小于 60 天, 因此在 GRACE 处理月 重力场数据时, 这些信号均为欠采样的, 消除它们的 短周期影响一般采用已知的潮汐和大气等模型, 但 是这些模型本身存在的误差会混叠入 30 天周期的重 力场估计中, 由此引起复杂的混频问题.

海洋潮汐的模型误差被认为是解算 GRACE 时 变重力场的最大误差来源(Han 等, 2004; Ray 和 Luthcke, 2006; Seo 等, 2008a; Visser 等, 2010). GRACE 连续变化的星下点轨迹使得上述问题更加严 重, 引起了重力场变化在时间和空间中的不均匀采 样. 不均匀采样导致了由海潮信号混叠引起的相当 复杂的重力场模型变化. 许多数据后处理方法被用 于减轻这种误差影响, 被称为去混频. 一般的去混频 方法是利用高斯滤波来平滑, 并利用去相关方法来去 除条纹影响. 然而, 用滤波的方法来消除这种影响的 效果是有限的, 有时甚至会引入不好的结果. 因此, 国外许多学者提出可以在任务设计时, 选择合适轨 道参数的卫星编队, 通过这种方式来研究是否可以 解决混频问题(Jekeli, 1996; Seo 等, 2008b; Zenner 等, 2010; Elsaka, 2010; Wiese 等, 2009, 2011).

在这一背景下, 本文利用包含各种不同方向观 测量的卫星编队, 通过仿真实验, 研究它们在重力场 反演中消除或削弱混频误差的影响的可行性, 并分 析比较了各种卫星编队在海潮混频误差影响下反演 重力场的能力.

\section{1 混频产生的原因及其对重力场解算的影响}

根据 Nyquist 采样定律, 只有当采样频率大于信 号中最高频率的 2 倍时, 采样之后的数字信号才能完 整保留原始信号中的信息，即不会产生混频.

对 GRACE 任务来说, 引起混频误差主要有如下 两个原因(赵倩等, 2011):

(1) GRACE 卫星运行状态(轨道)所造成的数据 欠采样, 导致重力场信号与其他信号发生混频. GRACE 卫星的轨道是一种自由下落的轨道, 由于地
球质量的时空变化、大气阻力等因素的影响, 不可能 保持同一个重复轨道不变, 即卫星轨道不严格重复, 根据这样的轨道所得到的数据是欠采样的, 以此来 确定和表达全球的球谐函数, 其中的混频现象将非 常复杂.

(2) 解算重力场时, 各种背景模型(海潮、大气等) 的不精确引起的混频. GRACE 卫星的主要目标是探 测由水文变化所引起的地球重力场的月、周年和长期 变化, 以及由其他地球物理现象所引起的周年和长 期变化 (如地震、冰后回弹、极地和 Greenland 冰雪 融化等). 为了达到这一科学目标, 在 GRACE 数据处 理中会利用先验模型扣除海潮、极潮以及大气和海洋 负荷所引起的地球重力场变化, 但是由于海潮、大气 和海洋负荷去混频模型存在误差, 导致其无法正确 描述相应地球物理信号的高频变化, 从而最终对 GRACE 重力场模型再次产生混频影响.

对于混频误差造成的影响, Watkins 等(2008)给 出了在各种仿真情况下 GRACE 重力场解算的结果, 从图 1 可以看出, 在当前的 GRACE 数据下, 其解算 误差远远大于由于海潮混频和大气混频所造成的影 响, 但当降低轨道高度或者使用更高精度的星间激 光测距系统时, 混频问题就不能被忽略了, 其误差远 远超过 GRACE数据本身的误差所造成的影响. Wiese 在 2011 年也做了相关仿真实验, 假设一对 GRACEtype 卫星在 $475 \mathrm{~km}$ 高度下, 星间距离为 $220 \mathrm{~km}$, 近 圆近极轨道上运行 30 天, 且在 30 天的飞行中保持着 严格的重复轨道周期. 与 Watkins 得到的结果类似, 在提高星间测距精度或者降低轨道高度的情况下，

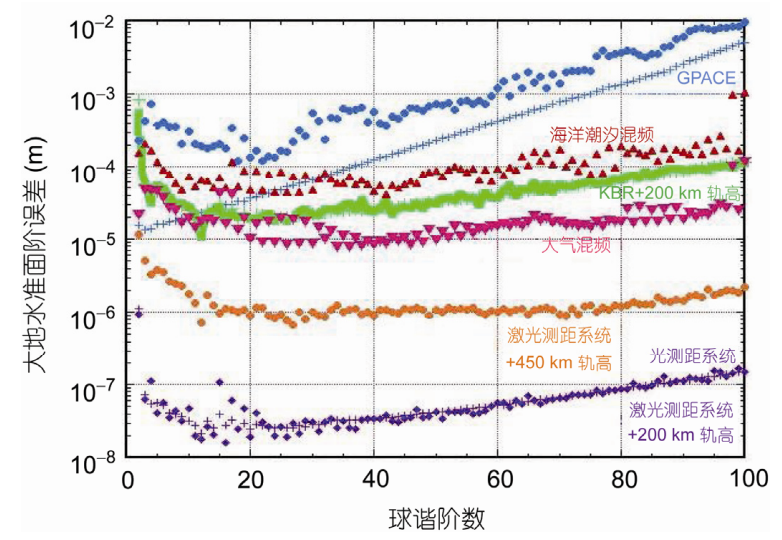

图 1 不同轨道高度和时变信号影响下大地水准面误差 据 Watkins 等(2008) 
各个时变地球物理信号对于重力场的解算影响很大. Loomis 等(2012)的研究也表明, 在当前地球物理模型 的精度下, 仅仅提高星间测距精度(如使用激光测距 系统)并不能立刻使重力场解算精度得到提高. 因此, 在末来的重力卫星任务, 必须采用适当的方法来消 除或削弱混频误差的影响.

\section{2 仿真实验}

\section{1 卫星编队模式和海潮模型}

在任务设计时, 可以尝试通过选择合适的轨道 参数和卫星编队模式来削弱混频误差的影响, 因为 不同的卫星编队模式包含着不同方向的观测量, 与 GRACE 任务单一的沿轨方向观测量相比, 对于重力 场解算结果会造成不同的影响. 这里所探讨的卫星 编队模式有三种: 串联编队(GRACE-type)、钟摆编队 (Pendulum-type)和车轮编队(n-s-Cartwheel-type)(Sneeuw 等, 2005).
三种卫星编队相对运动示意图如图 2, 这种运动 一般在相对运动坐标系中进行描述, 两颗卫星 $\mathrm{S}_{1}, \mathrm{~S}_{2}$, 坐标原点为其中一颗卫星质心, $x$ 轴在卫星运行轨道 面内沿速度方向, $y$ 轴指向卫星轨道面的正法线方向, $z$ 轴与 $x$ 和 $y$ 轴形成右手系. 后文也将 $x$ 轴方向称为 沿轨方向, $y$ 轴方向称为法向, $z$ 轴方向称为径向. 各 编队初始轨道参数如表 1 所示, 表 1 中标灰部分为各 卫星编队初始轨道根数的差异. GRACE-type 编队中 两颗卫星按照一定的前后顺序运行在同一轨道上, 只在 $x$ 轴沿轨方向有相对运动, 除了近地点角距 $\omega$ 和 平近点角 $M$ 有区别, 其他轨道参数均取为相同. 理论 上共面的 GRACE-type 卫星编队仅需要在平近点角 $M$ 的设置上有所不同即可, 但是通过仿真实验发现, 仅仅在平近点角有差异的两颗卫星, 其星间距离在 长时间内不能很好的保持在 $100 \mathrm{~km}$ 左右, 会有一个 线性减小的趋势. 为保证两颗卫星较长时间内的星 间距离变化较为稳定, GRACE B 卫星的初值是由第 一颗卫星的轨道内插而得到, 这样的初值选取可以
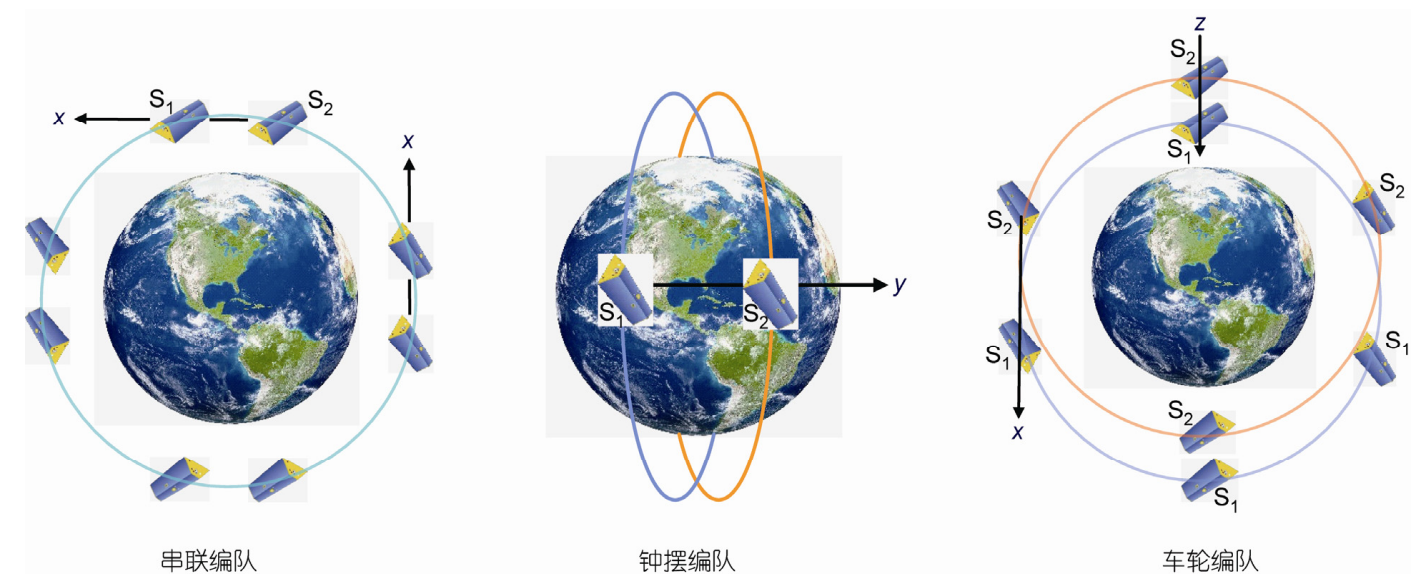

图 2 各种卫星编队相对运动示意图

表 1 各种卫星编队的初始开普勒轨道根数

\begin{tabular}{|c|c|c|c|c|c|c|c|c|c|}
\hline \multirow{2}{*}{ 轨道根数 } & \multicolumn{3}{|c|}{ GRACE } & \multicolumn{3}{|c|}{ Pendulun } & \multicolumn{3}{|c|}{ n-s-Cartwheel } \\
\hline & GRACE A & GRACE B & 差异 & Pen. A & Pen. B & 差异 & Cart.A & Cart.B & 差异 \\
\hline$a(\mathrm{~km})$ & 6728.137 & 6728.137 & $\Delta a=0.0$ & 6728.137 & 6728.137 & $\Delta a=0.0$ & 6728.137 & 6728.137 & $\Delta a=0.0$ \\
\hline$e$ & 0.001 & 0.001 & $\Delta e=0.0$ & 0.001 & 0.001 & $\Delta e=0.0$ & 0.0037 & 0.0037 & $\Delta e=0.0$ \\
\hline$i\left(^{\circ}\right)$ & 89.0 & 89.0 & $\Delta i=0.0$ & 89.0 & 89.0 & $\Delta i=0.0$ & 89.0 & 89.0 & $\Delta i=0.0$ \\
\hline$\Omega\left({ }^{\circ}\right)$ & 0.0 & 0.0 & $\Delta \Omega=0.0$ & 0.0 & 0.75 & $\Delta \Omega=0.75$ & 0.0 & 0.0 & $\Delta \Omega=0.0$ \\
\hline$\omega\left(^{\circ}\right)$ & 0.0 & 0.45 & $\Delta \omega=0.45$ & 0.0 & 0.0 & $\Delta \omega=0.0$ & 270 & 90 & $\Delta \omega=180$ \\
\hline$M\left(^{\circ}\right)$ & 0.0 & 0.4 & $\Delta M=0.4$ & 0.0 & 0.4 & $\Delta M=0.4$ & 90 & -90 & $\Delta M=180$ \\
\hline
\end{tabular}


很好的维持星间距离的稳定性. Pendulum 编队的两 颗卫星运行在不同的轨道平面上, 主要的观测量沿 $y$ 轴法线方向, 升交点赤经略有不同, 此处 $\Omega$ 值通过计 算得到，使得初始时刻的星间距离能保持在 $100 \mathrm{~km}$, 选取不同的 $M$ 值是为了使得星间距离在极点最小处 不要过小, 影响重力场解算, 同时使测量值中也可以 包含部分沿轨方向观测量. Cartwheel-type 编队中两 颗卫星运行在同一轨道平面上, 其中一颗卫星围绕 另一颗卫星星做长短半轴比为 $2: 1$ 的椭圆相对运动, 这种卫星编队可以同时提供 $x$ 轴沿轨方向和 $z$ 轴径向 两个方向的卫星观测量. 两颗卫星除了近地点角距 $\omega$ 和平近点角 $M$ 不同, 其他轨道根数均相同, 其中 $\omega$ 和 $M$ 的差异为 $\pi$. 一颗星的远地点和另一颗星的近地点 的距离为这两颗卫星在径向方向的最大距离, 而沿 轨方向的最大距离是径向距离的两倍. 由相对运动 的径向距离最大值出现的方向, 可将径向车轮编队 分为东西径向车轮编队和南北径向车轮编队, 这里 主要考虑的是南北径向 Cartwheel-type.

当前很难对现有的海潮模型精度给出定量的描 述, 因此往往采用两个不同机构发布的模型之差作 为模型误差, 并通过计算其对重力场结果的影响, 来 定量混频问题对重力场解算的影响. 这里给出用于 仿真实验中所采用的两种海潮模型 FES2004(Lyard 等, 2006)和 EOT08a(Savcenko 和 Bosch, 2008).

FES2004(Finite Element Solution 2004) 模型 (Lyard 等, 2006) 是根据潮汐流体动力学方程计算出 来, 并做了空间分辨率为 $0.125^{\circ} \times 0.125^{\circ}$ 的数据同化. FES2004 模型与以往的海潮模型如 CSR4.0(Center for Space Research version 4)或者 GOT00(Goddard Ocean Tide model 2000)相比, 具有更好的精度和分辨率, 因此很多数据处理中心如 CSR, GFZ 和 JPL 等在做 GRACE 重力场解算时都使用此模型来进行海潮改正.

EOT08a(Empirical Ocean Tide) 模型 (Bosch 和 Savcenko, 2008) 是一种包含主要潮汐分量的新的全 球潮汐模型, 使用经验分析的方法利用近 13 年(1992 年 10 月至 2005 年 10 月)的多代卫星测高数据得到, 空间分辨率为 $0.25^{\circ} \times 0.25^{\circ}$. 主要联合的测高卫星有 Topex/Poseidon, Jason-1, ERS-1, ERS-2, GFO 和 ENVISAT 等, 数据联合过程中也采用了很多预处理 手段.

这里并未就海潮模型的每一个分量的影响进行 详细分析, 海潮模型 FES2004 和 EOT08a 均采用其所
包含的全部海潮分量.

\section{2 仿真方案设计}

利用 2.1 节所给出的三种卫星编队, 设计了两个 方案, 方案一不考虑混频误差的影响, 方案二则考虑 海潮模型混频误差的影响. 两种方案中的观测量误 差均给定为: 轨道误差 $1 \mathrm{~cm}$, 星间距离变率误差 $10^{-8} \mathrm{~m} \mathrm{~s}^{-1}$, 各卫星编队均仿真 30 天的观测量来反演 重力场. 这里星间距离变率误差的量级是通过实验 给出的, 它对重力场反演造成的影响小于海潮模型 混频误差的影响(赵倩, 2012). 为了保证仿真方案的 真实性, 两种方案中同时考虑了大气以及陆地水等 时变信号的影响, 但在轨道积分和重力场反演阶段 均采用相同的模型, 大气模型为 ECMWF, 陆地水模 型为 GLDAS, 表 2 给出了两个方案中静态重力场和 各种时变信号模型的选择.

仿真平台均基于武汉大学自主研发的动力法定 轨和反演地球重力场软件. 仿真过程包括两部分, 同 时仿真轨道数据和星间观测数据, 并处理它们来做 重力场估计．第一部分使用一组真实模型 (truth models)来进行测量仿真; 第二部分则使用一组参考 模型(refer models)来进行仿真模拟. 真实模型和参考 模型之差反映了当前误差条件下模型的精度.

方案一没有考虑海潮模型及混频误差的影响, 在轨道积分和重力场反演阶段均使用了 FES2004 海 潮模型，所得到的结果仅受到不同静态重力场模型 和观测量误差的影响; 方案二则完全考虑了海潮模 型误差的影响, 在轨道积分和重力场反演阶段除了 海潮模型有所变化, 背景重力场和其他时变信号模 型均采用相同的模型，且其中加入的星间距离变率 误差的量级对重力场反演造成的影响小于海潮模型

表 2 各方案模型的选择

\begin{tabular}{ccc}
\hline & 轨道积分 & 重力场反演 \\
\hline 方案一 & 真实模型 & 仿真模型 \\
静态场 & EIGEN-GL05C & EGM96 \\
海洋潮汐 & FES2004 & FES2004 \\
大气 & ECMWF & ECMWF \\
际地水文 & GLDAS & GLDAS \\
\hline 方案二 & & \\
静态场 & EIGEN-GL05C & EIGEN-GL05C \\
海洋潮汐 & FES2004 & EOT08a \\
大气 & ECMWF & ECMWF \\
陆地水文 & GLDAS & GLDAS \\
\hline
\end{tabular}


混频误差的影响, 这时得到的重力场结果显示的是 海潮模型误差混频所造成的影响, 对于研究时变信 号影响下的重力场反演来说, 这是一种十分切合实 际的反演方案.

\section{3 结果比较分析}

图 3 给出了不同方案解算的大地水准面阶误差 的变化, 表 3 给出了不同方案得到的大地水准面阶误 差统计, 图 4 则给出了大地水准面阶误差的全球分布 情况, 为了更好的显示各卫星编队反演地球重力场 的误差分布形式, 图 4 中两种方案的调色板所表示的 误差量级不同.

无论是重力场解算的统计结果、大地水准面阶误 差变化还是误差的全球分布, 两种方案针对是否考 虑海潮模型混频误差的影响, 得到了不同的结果.

\subsection{1 方案一}

从表 3 所统计的大地水准面阶误差可以知道, 当 仅考虑静态背景场的影响而忽略海潮模型的混频误 差时, n-s-Cartwheel-type 编队解的 RMS 值最小, Pendulum 编队次之, GRACE 编队误差最大, 与 GRACE-type 编队相比, n-s-Cartwheel-type 编队对于 重力场反演精度的提高幅度可达 $43 \%$, 可见在这种

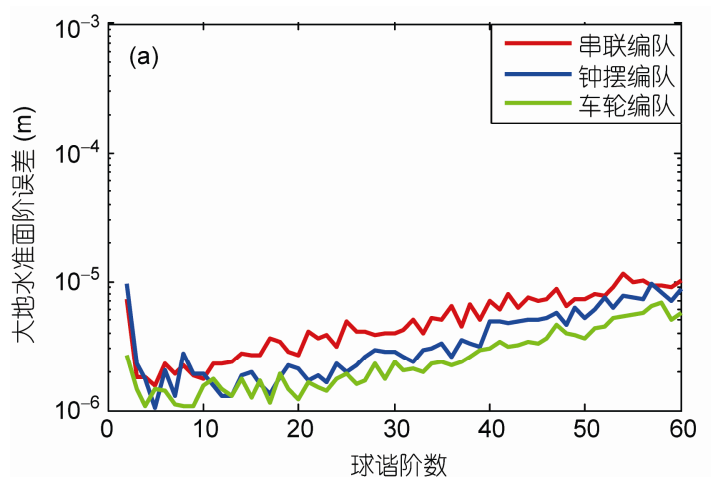

情况下利用卫星编队来反演重力场对其精度的提高 有很大好处.

图 3(a)和图 4((a) (c))给出了更加详细的重力场 解算信息. 所探讨的三种卫星编队中, GRACE-type 编队只包含沿轨方向观测量, Pendulum-type 编队同 时包含沿轨方向和法向观测量，而 Cartwheel-type 编 队有沿轨方向和径向观测量. 当不考虑海潮模型的 混频误差时, 无论从重力场各阶次的解算精度还是 重力场的误差全球分布上来看, GRACE-type 编队的 重力场解算结果最差，其他两种编队的结果几乎优 于其一个数量级, 这说明多方向的观测量有利于重 力场解算精度的提高; 同时, Cartwheel-type 编队的重 力场解算结果又较 Pendulum-type 编队稍好, 这说明 径向观测量是重力场信息的主要来源.

更细节的重力场信息也可以从大地水准面阶误 差的全球分布上得到. Cartwheel-type 编队的重力场 解算结果趋于各向同性, 而 GRACE-type 编队和 Pendulum-type 编队则表现出各向异性的敏感度, 造 成这种现象的原因在于, 车轮编队中均匀的包含了 沿轨方向和径向两个方向的观测量, 而串联编队只 包含一个方向(沿轨方向)观测量, 钟摆编队则由于轨 道设计的原因，使得其观测量中法向观测量占优，湮 没了沿轨方向观测量的贡献. 从这可以看出, 单一方

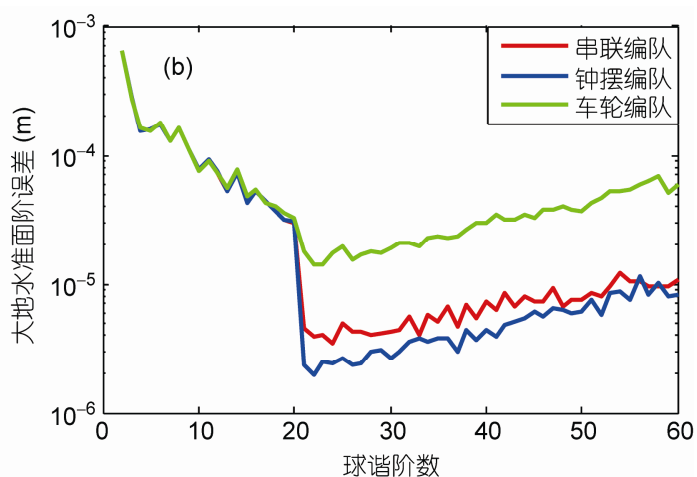

图 3 方案一 (a)和方案二(b)解算的大地水准面阶误差变化 $(n=60)$

表 3 不同方案得到的大地水准面阶误差统计(单位: mm)

\begin{tabular}{|c|c|c|c|c|c|c|}
\hline \multirow{2}{*}{ 编队类型 $(n=60)$} & \multicolumn{3}{|c|}{ 方案一 } & \multicolumn{3}{|c|}{ 方案二 } \\
\hline & RMS & 最小值 & 最大值 & RMS & 最小值 & 最大值 \\
\hline GRACE & 0.371 & -2.006 & 1.944 & 0.825 & -2.797 & 2.369 \\
\hline Pendulum & 0.333 & -1.399 & 1.426 & 0.825 & -2.804 & 2.306 \\
\hline n-s-Cartwheel & 0.211 & -0.959 & 1.058 & 0.848 & -2.971 & 2.541 \\
\hline
\end{tabular}




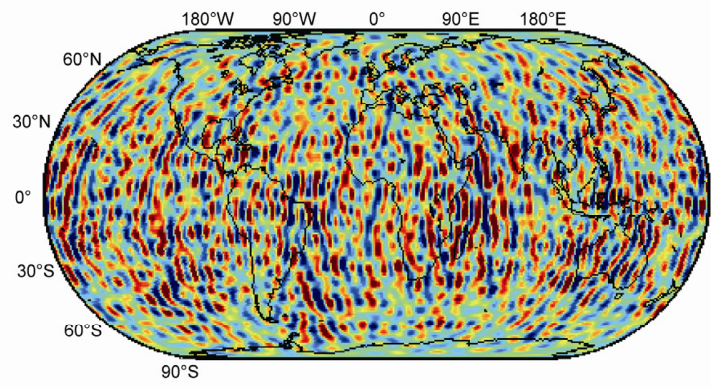

(a)

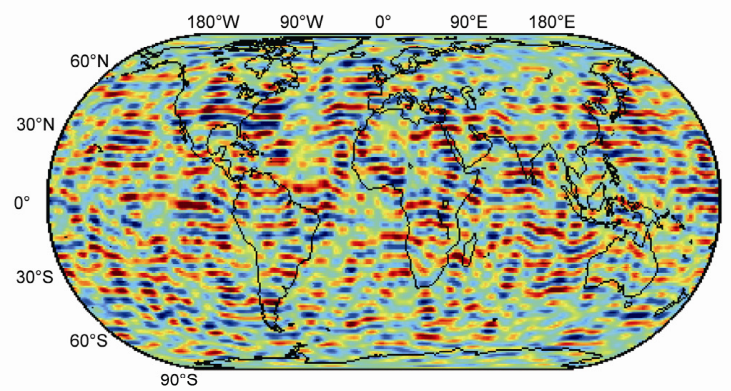

(b)

钟摆编队

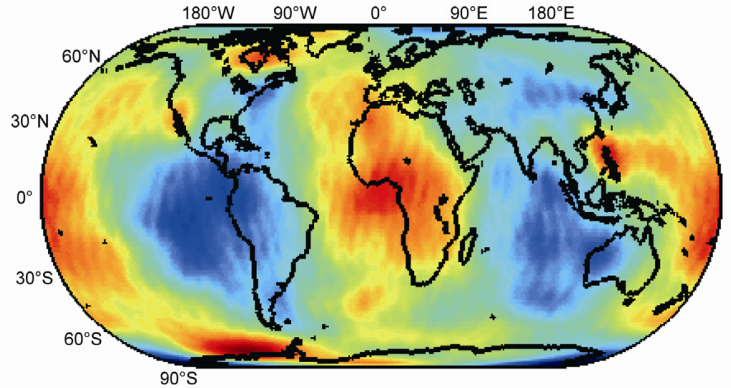

(d)

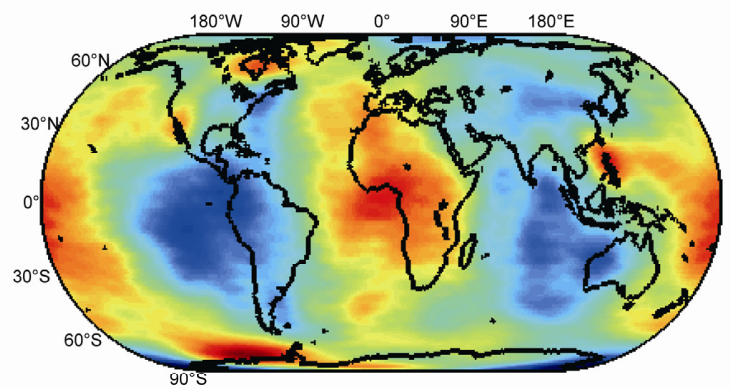

(e)

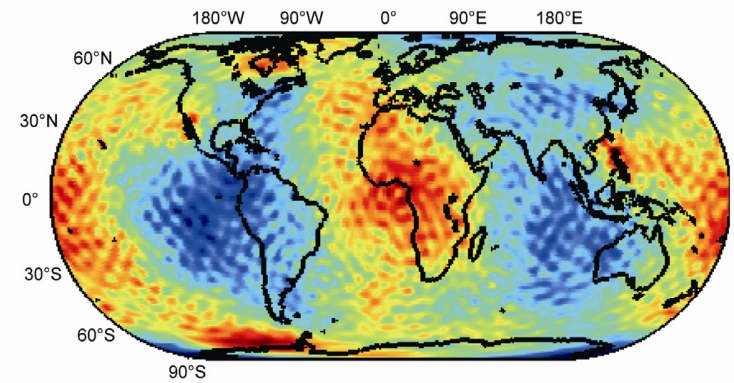

(f)

(c)

车轮编队

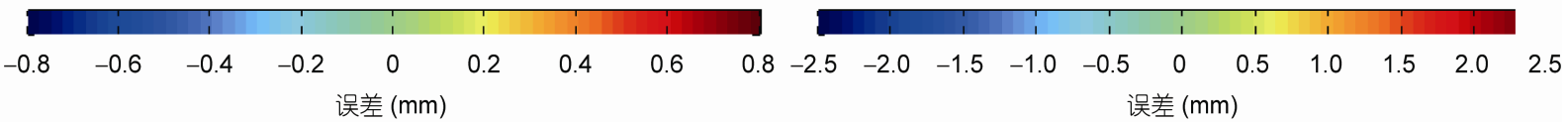

图 4 方案一 $((\mathrm{a}) \sim(\mathrm{c}))$ 和方案二 $((\mathrm{d}) \sim(\mathrm{f}))$ 解算的大地水准面阶误差的全球分布 $(n=60)$

向的重力场观测量(尤其是沿轨方向和法向的单一观 测量)是导致重力场各向异性敏感度的重要原因, 而 径向的观测量和均匀的多方向观测量联合可以大大 削弱这种影响.

\subsection{2 方案二}

当不考虑背景静态重力场的影响而加入海潮模
型造成混频误差时, GRACE-type 编队和 Pendulum 编 队的结果趋于一致, 而 n-s-Cartwheel-type 编队的精 度较差, 与方案一不考虑海潮模型混频误差的结果 大相径庭. 且重力场误差在低阶项时较方案一大了 1 2 个量级, 这说明海潮模型的混频误差会对重力场 低阶项产生较大影响.

考虑真实的海潮模型误差时, 海洋潮汐这一时 
变信号对 Cartwheel-type 编队的重力场结果影响最大, 其 RMS 值达到 $0.848 \mathrm{~mm}$, 精度最低. 只包含沿轨方 向观测量的 GRACE-type 编队和包含沿轨和法向观 测量的 Pendulum-type 编队的重力场解算的 RMS 值 精度相当. 对于包含沿轨和径向观测量的 Cartwheeltype 编队来说, 海潮信号模型误差的影响在中波处十 分显著, 尤其在 20 60 阶部分, 其误差超过了 GRACEtype 编队一个量级, 而几种编队在重力场长波处的影 响则显示出几乎相同的结果.

图 4((d) (f)) 显示的全球误差分布也可以看出, GRACE-type 和 Pendulum-type 编队仍然存在固有方 向的条纹误差, 而 Cartwheel-type 编队虽然消除了条 纹的影响, 但是其误差明显较另外两种编队大. 分析 出现这种情况的原因可能在于, Cartwheel-type 编队 的径向观测量对重力场的高频信号更为敏感, 因此 包含了更多的海潮模型误差的高频信号.

从以上分析可知, 当前海潮模型误差影响下, 即 使采用了更高精度的星间距离观测量, 并采用包含 多种方向观测量的卫星编队来反演地球重力场, 也 不能解决由海潮模型误差所导致的混频问题, 并且 由于 Cartwheel-type 编队的径向观测量对重力场高频 信号极为敏感, 导致其包含了更多的模型误差的高 频信号, 极大的影响了最终重力场反演的结果, 在混 频误差量级超过观测量误差时, 这种编队是不适合 于重力场反演任务的.

\section{3 结论}

随着新一代重力卫星任务的开展, 更高精度的
星间测量模式和无拖曳系统的实现, 如何消除或削 弱重力场解算中各种时变信号模型所造成的混频误 差成为了急需解决的问题. 本文通过大量仿真实验, 详细研究了在海洋潮汐时变信号的影响下, 利用各 种卫星编队反演地球重力场的结果, 探讨了利用卫星 编队消除混频误差并提高重力场解算精度的可行性.

结果表明, 在目前时变模型误差的影响下, 当星 间观测精度提高到一定量级, 即重力场解算中观测 量误差小于混频误差造成的影响时, 利用两颗卫星 构成的 Pendulum-type(沿轨及法向观测量)编队和 Cartwheel-type(沿轨及径向观测量)编队不能消除海 潮模型误差混频的影响, 得到更好的重力场解算结 果. 此时 Pendulum-type 和 GRACE-type 编队的重力 场解算精度相当, 而 Cartwheel-type 编队则在中短波 部分比这两种编队误差大了一个量级, 分析其原因 在于径向观测量对重力场的高阶变化更为敏感, 因 此包含了更多的海潮模型误差的高频信号. 在目前 情况下要削弱混频误差的影响, 需要采用更高时空 分辨率的方案, 要详如增加卫星的数目, 利用由多颗 卫星构成的卫星编队或卫星星座模式, 但这种方案 耗资巨大, 且需详细的仿真实验来验证其有效性. 此 外, 在混频误差造成的影响超过其他误差造成的影 响时，应避免采用包含径向观测量的卫星构形来进 行重力任务, 若其他误差的影响远大于混频误差的 影响, 或者在未来各种时变信号模型精度进一步精 化, 这时就可以考虑采用包含不同方向观测量的卫 星编队, 如 Cartwheel-type 编队来提高重力场的解算 精度.

\section{参考文献}

罗志才, 李琼, 张坤, 等. 2012. 利用 GRACE 时变重力场反演南极冰盖的质量变化趋势. 中国科学: 地球科学, 42: 1590-1596

赵倩, 姜卫平, 徐新禹, 等. 2011. GRACE 卫星重力场解算中混频误差影响的探讨. 大地测量与地球动力学, 31: 123-126

赵倩. 2012. 利用卫星编队探测地球重力场的方法研究与仿真分析. 博士学位论文. 武汉: 武汉大学

Chen J L, Wilson C R, Swo K W. 2006. Optimized smoothing of gravity recovery and climate experiment (GRACE) time-variable gravity observations. J Geophys Res, 111: B06408, doi: 10.1029/2005JB004064

Ditmar P, Teixeira da encarnacao J, Hashemi F H. 2011. Understanding data noise in gravity field recovery on the basis of inter-satellite ranging measurements acquired by the satellite gravimetry mission GRACE. J Geodesy, 86: 441-465

Elsaka B. 2010. Simulated satellite formation flights for detecting temporal variations of the earth's gravity field. Ph.D. Dissertation. Germary: University of Bonn

Han S C, Jekeli C, Shum C K. 2004. Time-variable aliasing effects of ocean tides, atmosphere, and continental water mass on monthly mean GRACE gravity field. J Geophys Res, 109: B04403, doi: 10.1029/2003JB002501

Jekeli C. 1996. Spherical harmonic analysis aliasing and filtering. J Geodesy, 70: 214-223 
Kim J. 2000. Simulation Study of A Low-Low Satellite-to-Satellite Tracking Mission. Doctoral Dissertation. Austin: University of Texas Klees R, Revtova E A, Gunter B C, et al. 2008. The design of an optimal filter for monthly GRACE gravity models. Geophys J Int, 175: 417-432 Kurtenbach E, Mayer-Gurr T, Eicker A. 2009. Deriving daily snapshots of the Earth's gravity field from GRACE L1B data using Kalman filtering. Geophys Res Lett, 36: L17102, doi: 10.1029/2009GL039564

Loomis B D, Nerem R S, Luthcke S B. 2012. Simulation study of a follow-on gravity mission to GRACE. J Geodesy, 86: 319-335

Lyard F, Lefevre F, Letellier T, et al. 2006. Modelling the global ocean tides: Modern insights from FES2004. Ocean Dyn, 56: 394-415

Ray R D, Luthcke S B. 2006. Tide model errors and GRACE gravimetry: Towards a more realistic assessment. Geophys J Int, 167: 1055-1059

Savcenko R, Bosch W. 2008. EOT08a-empirical ocean tide model from multi-mission satellite altimetry. Report No.81. Deutsches Geodatisches Forschungsinstitut (DGFI), Munchen, Germany

Seo K W, Wilson C R, Han S C, et al. 2008a. Gravity recovery and climate experiment (GRACE) alias error from ocean tides. J Geophys Res, 113: B03405, doi: 10.1029/ 2006JB004747

Seo K W, Wilson C R, Chen J L, et al. 2008b. GRACE's spatial aliasing error. Geophys J Int, 172: 41-48

Sneeuw N, Flury J, Rummel R. 2005. Science requirements on future missions and simulated mission scenarios. Earth Moon Planets, 94: $113-142$

Tapley B D, Bettadpur S, Ries J C, et al. 2004. GRACE measurements of mass variability in the Earth system. Science, 305: 503-505

Visser P, Sneeuw N, Reubelt T, et al. 2010. Space-borne gravimetric satellite constellations and ocean tides: Aliasing effects. Geophys J Int, 181: 789-805

Wahr J, Molenaar M, Bryan F. 1998. Time variablility of the Earth's gravity field: Hydrological and oceanic effects and their possible detection using GRACE. J Geophys Res, 103: 30205-30229

Wahr J, Swenson S, Zlotnicki V, et al. 2004. Time-variable gravity from GRACE: First results. Geophys Res Lett, 31: L11501, doi: 10.1029/2004GL019779

Watkins M, Sprague G, Case K, et al. 2008. Time Variable Gravity Mapping Mission (Grace Follow-On/Grace II) Study. In: GRACE Science Team Meeting. 237-250

Wiese D N, Folkner W M , Nerem R S. 2009. Alternative mission architectures for a gravity recovery satellite mission. J Geodesy, 83: 569-581

Wiese D N, Visser P, Nerem R S. 2011. Estimating low resolution gravity fields at short time intervals to reduce temporal aliasing errors. Adv Space Res, 48: 1094-1107

Zenner L, Gruber T, Jäggi A, et al. 2010. Propogation of atmospheric model errors to gravity potential harmonics-impact on GRACE de-aliasing. Geophys J Int, 182: 797-807 